\title{
Regulation of microRNAs by epigenetics and their interplay involved in cancer
}

\author{
Xiaolan Liu' $1,2,3,4$, Xiaoyan Chen ${ }^{1,2,3,4}$, Xinfang Yu ${ }^{1,2,3,4}$, Yongguang Tao ${ }^{1,2,3,4}$, Ann M Bode ${ }^{5}$, Zigang Dong ${ }^{5}$ \\ and $\mathrm{Ya} \mathrm{CaO}^{1,2,3,4^{*}}$
}

\begin{abstract}
Similar to protein-coding genes, miRNAs are also susceptible to epigenetic modulation. Although numerous miRNAs have been shown to be affected by DNA methylation, the regulatory mechanism of histone modification on miRNA is not adequately understood. EZH2 and HDACs were recently identified as critical histone modifiers of deregulated miRNAs in cancer and can be recruited to a miRNA promoter by transcription factors such as MYC. Because miRNAs can modulate epigenetic architecture and can be regulated by epigenetic alteration, they could reasonably play an important role in mediating the crosstalk between epigenetic regulators. The complicated network between miRNAs and epigenetic machineries underlies the epigenetic-miRNA regulatory pathway, which is important in monitoring gene expression profiles. Regulation of miRNAs by inducing epigenetic changes reveals promising avenues for the design of innovative strategies in the fight against human cancer.
\end{abstract}

Keywords: microRNA, Epigenetic, DNA methylation, Histone modification, Cancer

\section{Introduction}

MicroRNAs (miRNAs) are endogenous non-coding RNAs ( 22 nucleotides) that regulate gene expression at the post-transcriptional level. Similar to protein-coding genes, the expression of miRNAs is also regulated by both genetic and epigenetic mechanisms. Approximately half of the miRNA genes are located in fragile regions of the genome that are associated with deletion, duplication or translocation. This suggests that alterations in miRNA genes could be a more general defect in tumor cells [1]. With the recent discovery of epigenetic processes, an increasing number of miRNAs have been discovered to be affected by epigenetic aberrations in tumor cells [2]. Clearly, miRNA genes can be epigenetically regulated by DNA methylation and/or histone modifications. In turn, a subgroup of miRNAs, named epi-miRNAs, was recognized to directly target enzymatic effectors involved in epigenetic modulation [3]. These observations suggest the existence of a regulatory circuit between epigenetic modulation and miRNAs,

\footnotetext{
* Correspondence: ycao98@vip.sina.com

${ }^{1}$ Cancer Research Institute, Central South University, Changsha, Hunan

410078, China

${ }^{2}$ Key Laboratory of Carcinogenesis and Cancer Invasion, Ministry of

Education, Changsha, Hunan 410078, China

Full list of author information is available at the end of the article
}

which could have a significant effect on transcription [4]. Because miRNAs have a large impact on carcinogenesis through the regulation of diverse target genes, understanding the regulatory mechanisms of miRNA expression is important in treatment and prevention of human cancers.

Epigenetic changes such as DNA methylation and histone modification are associated with chromatin remodeling and regulation of gene expression in mammalian development and human diseases, including cancer. The first evidence for the epigenetic regulation of miRNAs in cancer was obtained by using chromatin modifying drugs to reactivate miRNAs at the transcriptional level [5]. Emerging evidence shows that more than one hundred miRNAs are regulated by epigenetic mechanisms, and about onehalf of them are modulated by DNA methylation [6]. Because $\mathrm{CpG}$ methylation can be analyzed by a variety of techniques with relatively high sensitivity, we can identify miRNAs deregulated by aberrant DNA methylation in primary samples that might be limited in number and of poor quality [7]. However, DNA methylation does not always take place alone, but often occurs in the presence of other epigenetic modifications, such as histone modification, which constitutes the second major epigenetic regulatory system of miRNAs. While DNA methylation leads to miRNA silencing, histone modification, especially 
histone methylation, can either trigger or suppress miRNA expression, depending on the target amino acid residues and the extent of methylation. Given that miRNA expression is tissue-specific and depends on cellular context, histone modification might regulate distinct subpopulations of miRNAs in different types of cancers. In addition, the analysis of chromatin modification status should be performed on pure cell populations. Accordingly, identifying the specific miRNAs, which are regulated by aberrant histone modification in clinical tissue samples, remains challenging [8]. For the above reasons, the role of histone modification in miRNA deregulation is still obscure and has been poorly elucidated thus far.

In order to systematically understand the regulatory mechanism of histone modification on miRNA expression, in this review we will focus on the current knowledge regarding the miRNAs deregulated by epigenetic mechanisms, especially histone modification, in human cancer. We will also connect the indirect crosstalk between epigenetic regulators through miRNA mediation.

\section{Epigenetic mechanisms of miRNA dysregulation in cancer}

With the progress in DNA methylation detection techniques, numerous miRNAs have been identified that are modulated by DNA methylation, shedding light on the epigenetically regulated miRNAs. Among them, miR-9, miR-148, miR-124, miR-137, miR-34, miR-127 and miR512 reportedly can be silenced by CPG hypermethylation in at least three types of cancers [6]. However, it is still largely unknown which miRNAs can be altered owing to histone modifications. To date, histone methylation and histone deacetylation were confirmed to be involved in miRNA regulation. Understanding which and how miRNAs are regulated by histone modifying effectors in cancer might be helpful in tumor treatment.

\section{MiR-29}

The miR-29 family, which targets DNA methyltransferase 3 (DNMT3), is the first reported epi-miRNA, and is also the most extensively studied miRNA that is regulated by histone modification [9]. Recent studies show that transcription factors can regulate miRNA expression through epigenetic mechanisms. For instance, MYC can induce epigenetic regulation of miR-29 repression through histone deacetylation and tri-methylation in B-cell lymphomas (BCL), since it can recruit histone deacetylase 3 (HDAC3) and enhancer of zeste homolog 2 (EZH2) to the miR-29 promoter, forming a MYC/HDAC3/EZH2 corepressor complex. Without $\mathrm{MYC}$, however, the lack of binding of HDAC3 and EZH2 to the miR-29 promoter results in increased miR-29 expression [10]. Therefore, MYC plays an indispensable role in the epigenetic repression of miR-29 by inducing histone deacetylation and histone tri- methylation. Meanwhile, EZH2 can also repress miR-494 to create a positive feedback loop, which in turn increases MYC abundance and then sustains miR-29 repression in BCL [10]. These properties indicate that different epigenetic modifications can cooperatively regulate the same miRNA, whereas a specific epigenetic effector can regulate more than one miRNAs in the same type of tumor.

Previous research evidence suggested that the transcription factor Yin and yang 1 (YY-1) can recruit various proteins such as EZH2 and HDACs to target genes during various epigenetic events [11-13]. Later Wang et al. confirmed that nuclear factor $\kappa B(N F-\kappa B)$ up-regulated YY-1 resulted in the recruitment of EZH2 and HDAC1 to the miR-29 promoter in myoblasts, leading to the downregulation of miR-29 and maintaining cells in an undifferentiated state. Once myogenesis starts, the repressive complex containing YY-1/EZH2/HDAC will be replaced by an activating complex. Therefore, miR-29 is restored and in turn targets YY1 to ensure differentiation. However, the constitutively activated NF- $\mathrm{KB}-\mathrm{YY} 1$ in rhabdomyosarcoma (RMS) could induce epigenetic repression of miR-29 and thereby block differentiation. The inhibition of NF- $\kappa$ B activity by inhibitor of nuclear factor $\kappa B \alpha(\mathrm{I} \kappa \mathrm{B} \alpha)$ would remarkably decrease the level of YY1, and consequently neither EZH2 nor HDAC1 could be recruited to miR-29 promoter [14]. This study demonstrated that NF$\mathrm{\kappa B}$ might be an upstream regulator of the epigenetic status of miR-29 in skeletal myogenesis.

In addition to these effects in solid tumors, miR-29 deregulation by epigenetic mechanisms can also be found in human hematological cancers. For instance, in acute myeloid leukemia (AML), the transcriptional complex NF$\kappa \mathrm{B} / \mathrm{Sp} 1$ can interact with HDAC1 and HDAC3 to form the NF-kB/Sp1/HDAC complex on miR-29b enhancer, which resulted in the silencing of miR-29b. Notably, MYC can directly bind to miR-29b promoter and stimulate the activity of NF-kB/Sp1/HDAC. Therefore, the down-regulation of miR-29b is MYC-dependent [15]. Interestingly, HDAC inhibition could restore the expression of miR-29b in only one third of chronic lymphocytic leukemia (CLL) samples [16]. For the other two-thirds of CLL cases, the identification of other histone modifications that contribute to epigenetic silencing of miR-29b still needs to be accomplished.

In summary, binding of MYC or NF- $\mathrm{kB}$ on the miR-29 promoter seems to be a primary event in miR-29 silencing, and thereby induces the initial step of its chromatin modification. Subsequently, various histone modifying enzymes such as EZH2 and HDACs can be recruited to the miR-29b promoter. These enzymatic effectors might receive signals from their initiator, and then function as an executor of this epigenetic event. Additionally, the transcription factors YY1 and Sp1, which are dispensable in this regulation, might act as bridges that connect the initiator and the executor. 


\section{Let-7 family}

Reportedly, the let-7 miRNAs, which target oncogenic Ras and function as tumor suppressors, are located in fragile genomic regions that are frequently deleted in human cancers $[1,17]$. Besides genomic alterations, the let- 7 genes could also be regulated by epigenetic mechanisms. MYC induced by $H$. pylori CagA in gastric cancer cells can suppress the expression of let-7a and let-7c through two epigenetic approaches: (1) MYC stimulates EZH2 expression by reducing its negative regulators, miR-26a and miR101; (2) MYC interacts with DNMT3B and EZH2 on the let-7 promoter, and consequently the let-7 gene is silenced through both DNA and histone methylation. Accordingly, the Ras pathway is activated to contribute to carcinogenesis [18]. However, in human lung cancers, let-7a-3 was found to be hypomethylated, which is different from its status in normal lung tissues [19], suggesting that differential, and even opposite, epigenetic regulations might take place in the same miRNA according to the cell context. In view of that, exploration into the epigenetic modulation of the let-7 gene family is essential.

\section{MiR-15a/miR-16 cluster}

The miR-15a and miR-16 are located on the human chromosome 13q14, and consequently their levels could be lowered by deletions in 13q14, which occur commonly in CLL and mantle cell lymphoma (MCL) [20]. However, down-regulation of these two miRNAs is also observed in many CLL cases with intact chromosome 13 [21], indicating that other mechanisms might be involved in this regulation. Recently, HDAC inhibition was proposed to trigger the expression of miR-15a and miR-16 in some CLL samples, suggesting they could be epigenetically silenced by histone deacetylation [16]. Interestingly, Zhang et al. revealed that MYC repressed miR-15a/16-1 cluster expression through recruitment of HDAC3 in MCL [22], emphasizing that MYC plays an important role also in the epigenetic silencing of the miR-15a/miR-16 cluster.

\section{MiR-31}

Like the miR-15a/miR-16 cluster, miR-31 is also considered to be both genetically and epigenetically regulated. Genetic loss of miR-31, which resides in the deletion hotspot 9p21.3, was demonstrated to be beneficial for tumor progression and was observed in several types of human cancers [23]. However, the loss of miR-31 expression can also be detected in tumor cells without 9p21.3 deletion. DNA methylation and/or EZH2-mediated histone methylation were recently confirmed to contribute to miR-31 loss in melanoma, breast cancer and adult $\mathrm{T}$ cell leukemia (ATL) [24-26]. Also ChIP-PCR assay results revealed the YY1 binding motifs around the miR-31 region, which recruit EZH2 and mediate epigenetic silencing of miR-31. Although YY1 could contribute to miR-31 repression, knockdown of YY1 in ATL cells without genetic deletion only restored a small proportion of the silenced miR-31 and could not remove EZH2 completely from the miR-31 region [26]. Thus, YY1 does not appear to be indispensable in EZH2-mediated miR-31 silencing, pointing out the existence of other important upstream regulators.

\section{MiR-23a}

MiR-23a was demonstrated to be transcriptionally repressed by MYC in many cancer cells [27]. Besides MYC, other transcription factors can also epigenetically regulate miR-23a expression. For instance, the NF-kB p65 subunit can recruit HDAC4 to miR-23a promoter, thereby silencing the expression of miR-23a in human leukemic Jurkat cells [28]. HDAC4 as a member of class IIa HDACs is expressed tissue-specifically in heart, smooth muscle and brain [29]. Thus, compared with the widely expressed class I HDAC enzymes (HDAC1, $-2,-3$, and -8), HDAC4 seems to have a tissue-restricted role in epigenetic regulation of miRNAs.

\section{Other down-regulated miRNAs}

In addition to the above miRNAs, multiple miRNAs that are downregulated by histone modifications also exist. For instance, miR-139-5p, miR-125b, miR-101, let-7c, miR-200b were found to be epigenetically repressed by EZH2, and miR-449 was repressed by HDACs in human hepatocellular carcinoma (HCC) [30,31]. Similarly, EZH2 suppressed the expression of miR-181a, miR-181b, miR200b, miR-200c, let-7 and miR-203 in prostate cancer [32,33]. Moreover, the histone demethylase Jarid1b could also repress let-7e as well as miR-1246, miR-1826, and miR-361-5p by removing the active mark H3K4me3 in breast cancer [34]. However, the underlying molecular mechanisms of these miRNAs are still unknown and should be studied in detail.

\section{Up-regulated miRNAs}

Most of the miRNAs deregulated by aberrant patterns of histone modification in cancer cells are silenced, but some miRNAs, such as miR-224, miR-615 and miR-155, are activated by histone modification.

The miR-224 is the most significantly upregulated miRNA in HCC and was found to target apoptosis inhibitor-5 (API-5) to promote tumorigenesis [35]. However, the regulatory mechanism of miR-224 in liver disease is mostly obscure. Actually, miR-224 overexpression can be attributed to histone acetylation rather than genomic amplification or DNA hypomethylation. The histone acetylase protein EP300 acts as a positive regulator in this regulation, whereas HDACs function as negative regulators [36]. Considering that miR-224 overexpression could not be totally attenuated by inhibition of histone acetylation, other factors might also contribute to 
miR-224 upregulation. Similarly, a study in prostate cancer cells identified miR-615 as an epigenetically activated miRNA by DNA methylation loss and H3K9 acetylation gain [37].

As an oncogenic miRNA, miR-155 is overexpressed in many cancers such as breast cancer $[38,39]$. Recently, miR-155 in normal breast tissues was proposed to be epigenetically repressed by wild-type BRCA1, which interacted with $\mathrm{HDAC} 2$ to deacetylate $\mathrm{H} 2 \mathrm{~A}$ and $\mathrm{H} 3$ on the miR-155 promoter. In BRCA1-deficient or BRCA1-mutant cancer cells, however, the loss or mutation of BRCA1 resulted in miR-155 upregulation, since HDAC2 could not be recruited to the miR-155 promoter [40].

The regulatory models of miR-29 and other miRNAs suggest that the well-known transcription factor MYC, which is one of the most commonly overexpressed oncogenes in cancer, has some functions in the aspect of epigenetic regulation (Figure 1).

\section{The crosstalk between epigenetic regulators}

The importance of inhibitory signals that contribute to epigenetic gene silencing, especially DNA methylation and histone deacetylation, has been increasingly recognized in recent years. However, the crosstalk between these epigenetic regulators is not fully understood, because of the difficulty to apply a unique model that can explain DNA and histone modification in specific epigenetic events. Nevertheless, accumulating data support the existence of a circulation loop between DNA methylation and histone modification, i.e., DNA methylation directs histone modification and histone modification recruits more DNA methylation. All of these observations suggest a reciprocal crosstalk between DNA methylation and histone modification. Indeed, these epigenetic regulators can communicate and benefit each other to reinforce epigenetic gene silencing. In this scenario, miRNAs are becoming a crucial factor in the faithful transmission of different patterns of epigenetic modulation (Figure 2).

The miR-26a can be silenced by DNMTs in prostate cancer, which induces the accumulation of its target gene EZH2 and changes the global DNA methylation status [41], supporting the idea that miRNAs can mediate the interplay between epigenetic regulators. The miR-137 is another important mediator, which is silenced by promoter CpG island hypermethylation and targets lysine-specific demethylase 1 (LSD1) in colorectal adenomas [42]. Because LSD1 can stabilize DNMT1, a positive feedback loop exists between them. Besides the crosstalk between DNA and histone methylation, indirect crosstalk between DNA methylation and histone deacetylation also occur through miRNA mediation, such as miR-1 and miR-29. The miR-1, which targets HDAC4, is down-regulated in human HCC cells because of its CGI hypermethylation by DNMT1, thereby promoting the expression of HDAC4 [43]. Likewise, HDACs can induce miR-29 silencing in acute myeloid leukemia (AML), which in turn increases the expression of its target gene DNMT3 [15,44].

These findings indicate that epigenetic information can flow from one modulation to a miRNA, and then from the miRNA to another epigenetic pattern. As a member of epigenetic machinery, miRNAs can also contribute to the conversation between other epigenetic events.

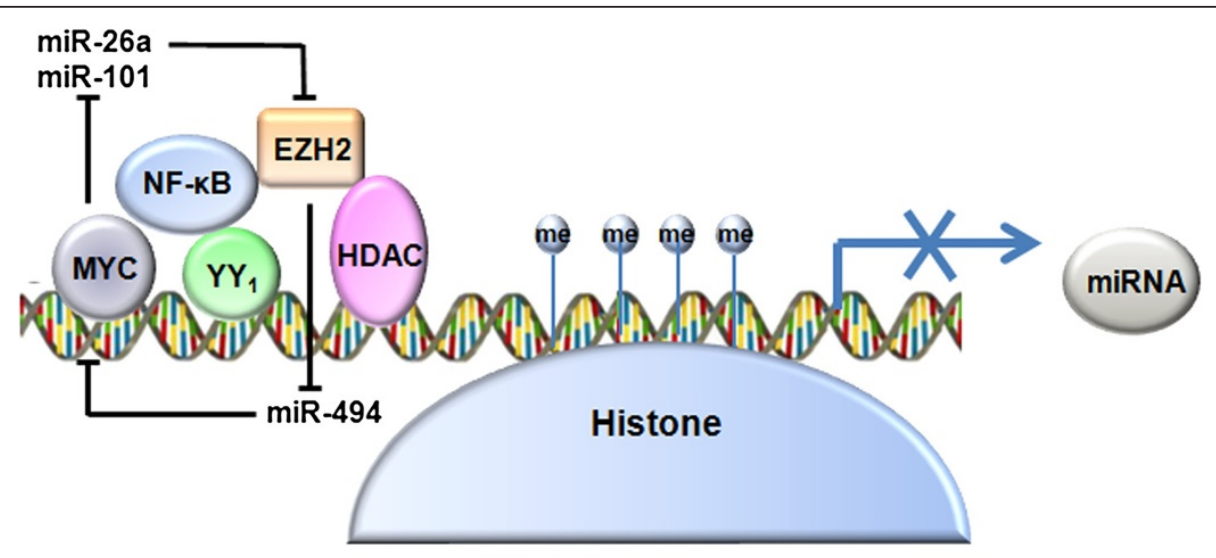

\section{me:histone methylation}

Figure 1 A model depicting the mechanisms of histone modification that repress miRNA expression. MYC or NF- $\mathrm{kB}$, which interacts with transcription factor $Y Y 1$ or Sp1 on miRNA promoter, is hypothesized to be the upstream regulator of miRNA silencing. Various histone modifying enzymes such as EZH2 and HDACs can be recruited to methylate and deacetylate histones. A positive feedback loop exists between MYC and EZH2: MYC stimulates EZH2 expression by reducing its negative regulators, miR-26a and miR-101; EZH2 can also increase the abundance of MYC by repressing miR-494. 


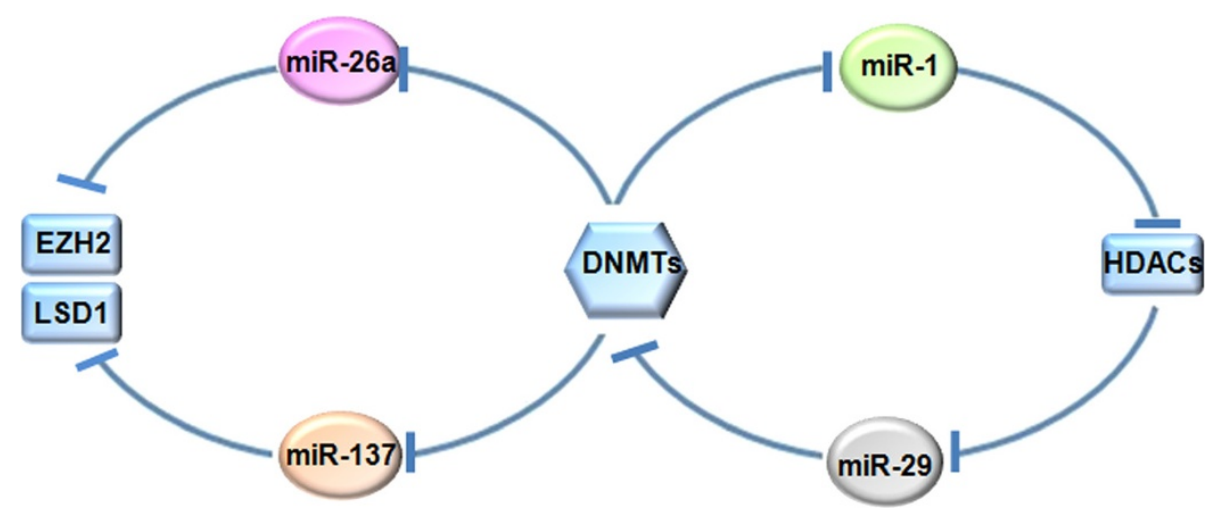

Figure 2 The role of miRNAs in mediating the crosstalk between epigenetic regulators. DNMT1 contributes to miR-1 silencing in HCC cells, thereby promoting the accumulation of its target HDAC4. The miR-29, which targets DNMT3, is down-regulated by HDACs in AML. Likewise, miR-26a and miR-137 are silenced by promoter CpG island hypermethylation, which induces the up-regulation of the target gene LSD1 in colorectal adenomas and $\mathrm{EZ} \mathrm{H} 2$ in prostate cancer.

\section{Controlling miRNA expression with epigenetic drugs}

The frequent dysregulation of miRNAs and their interplay with epigenetic regulators in cancer make them attractive biomarkers and prospective therapeutic targets in clinical applications. The therapeutic application of miRNAs in cancer involves two strategies: 1) inhibition of oncogenic miRNAs by using miRNA antagonists, such as anti-miRs or antagomiRs; or 2) introduction of tumor suppressor miRNAs through either synthetic miRNA mimics or by stable and vector-based transfection of genes coding for miRNAs [45]. Moreover, a deeper understanding of these epigenetically regulated miRNAs might lead to a novel therapeutic strategy based on using of epigenetic drugs to control the expression of both onco-miRNAs and tumorsuppressor-miRNAs for prevention or treatment of human cancers.

The abnormalities of epigenetic in cancer, unlike genetic lesions, can be reversed by epigenetic-regulated drugs, which provides an opportunity for epigenetic therapy. The goal of epigenetic therapy would be to target the chromatin in rapidly dividing tumor cells in order to bring them to a more 'normal state', while only mildly disturbing the epigenome of healthy cells [46]. Five kinds of epigenetic drugs are known, including DNMT inhibitors, HDAC inhibitors, histone acetyltransferase (HAT) inhibitors, histone methyltransferase (HMT) inhibitors and histone demethylase (HDT) inhibitors [47]. Most of the research efforts focused on the first two agent types. For example, two DNMT inhibitors, 5-azacytidine (5-AzaC) and 5-aza-2'-deoxycytidine (5-Aza-CdR), were approved by FDA to treat myelodysplastic syndromes (MDS) and AML [48]. In 2006, the FDA first approved the HDAC inhibitor suberoylanilide hydroxamic acid (SAHA) to treat cutaneous T-cell lymphoma (CTCL) [49]. Probably, with the discovery and elucidation of epigenetic-miRNA regulatory pathways, at least part of the observed therapeutic effects of these epigenetic agents, such as 5 -Aza-CdR, might be attributed to their effect on miRNAs.

The deregulated miRNAs that can be controlled by epigenetic drugs in human cancers are shown in Table 1. These agents can either cause the re-expression of silenced tumor suppressor miRNAs or repress oncogenic miRNAs that are over- expressed in cancer cells. Besides the most commonly used DNMT inhibitors and HDAC inhibitors, C646 is a novel HAT inhibitor that is able to inhibit histone acetyltransferase EP300 and suppress the upregulated miR-224 [36]. However, these drugs might work better together than individually. For example, the combined use of 3-deazaneplanocin A (DZNep) and trichostatin A (TSA), but not their single use, could dramatically induce miR-449 expression [50]. One possible reason for this activity is that miRNA genes are regulated by multiple epigenetic effectors, and thus inhibition of one factor might not reverse miRNA expression completely. Consequently, the idea of combining different types of epigenetic drugs to effectively control abnormal miRNA expression in cancer cells turns out to be quite exciting and attractive.

\section{Conclusion}

The connection between DNA methylation and histone modification has been described in several physiological systems and disease states. Recent investigations also added miRNAs to this complicated crosstalk [65], thereby reinforcing the effects of epigenetic reprogramming. A better understanding of epigenetic regulatory mechanism of miRNA expression will help to elucidate the complex network of epigenetic modifications and design innovative strategies for cancer treatment. Although DNA methylation is the best-studied epigenetic mechanism for miRNA deregulation, it is still largely unknown which miRNAs are 
Table 1 The deregulated miRNAs that can be controlled by epigenetic drugs in human cancers

\begin{tabular}{|c|c|c|c|c|c|}
\hline $\begin{array}{l}\text { Epigenetically } \\
\text { regulated miRNAs }\end{array}$ & Target protein & Cancer type & Epigenetic drug & Fold change & Citation \\
\hline \multicolumn{6}{|l|}{ Down-regulated } \\
\hline \multirow[t]{2}{*}{ miR-449 } & \multirow[t]{2}{*}{ CDK6, CDC25A, C-MET } & Breast cancer & DZNep + TSA & $>20$ folds & {$[50]$} \\
\hline & & Hepatocellular carcinoma & TSA & $10-30$ folds & {$[31]$} \\
\hline miR-125b & PIGF & Hepatocellular carcinoma & 5-Aza-CdR & $>2$ folds & [51] \\
\hline miR-126 & VEGFA, PIK3R2 & Bladder cancer & 5-Aza-CdR + PBA & $>10$ folds & {$[52]$} \\
\hline miR-127 & $\mathrm{BCL6}$ & $\begin{array}{l}\text { Bladder cancer and clear cell } \\
\text { renal cell carcinomas }\end{array}$ & 5-Aza-CdR + PBA/TSA & $>10$ folds & {$[5,53]$} \\
\hline miR-1-1 & FOXP1, MET, HDAC4 & Hepatocellular carcinoma & 5-AzaC & $>20$ folds & [43] \\
\hline miR-34 & C-MYC, CDK6, & $\begin{array}{l}\text { Lung cancer, melanomas } \\
\text { and prostate cancer }\end{array}$ & 5-Aza-CdR & $>10$ folds & {$[54,55]$} \\
\hline $\begin{array}{l}\text { miR-181a,b, miR-200a,b,c, } \\
\text { and miR-203 }\end{array}$ & BMI1 and RING2 & Prostate cancer & DZNep/5-Aza-CdR/SAHA & $2-20$ folds & {$[32]$} \\
\hline \multirow[t]{2}{*}{ miR-31 } & \multirow{2}{*}{$\begin{array}{l}\text { SRC, RAB27a, NIK and MET, } \\
\text { RhoA and WAVE3 }\end{array}$} & Melanoma & 5-Aza-CdR/DZNep & $>2$ folds & [24] \\
\hline & & Breast cancer & 5-Aza-CdR or 5-Aza-CdR + TSA & $>5$ folds & {$[25]$} \\
\hline \multirow[t]{2}{*}{ miR-29 } & \multirow{2}{*}{$\begin{array}{l}\text { Mcl-1, DNMT3A, DNMT3B, } \\
\text { SP1, TCl-1, CDK6 and IGR1F }\end{array}$} & Aggressive B-Cell Lymphomas & DZNep + SAHA & 2-10 folds & [10] \\
\hline & & $\begin{array}{l}\text { Chronic lymphocytic leukemia } \\
\text { and acute myeloid leukemia }\end{array}$ & LBH589/SAHA/AR-42 & $2-5$ fold & {$[15,16]$} \\
\hline miR-15a/miR-16 & $\mathrm{BCL}-2$ and $\mathrm{MCL}-1$ & Chronic lymphocytic leukemia & LBH589/SAHA & $2-5$ fold & {$[16]$} \\
\hline miR-205 & SIP1 and ZEP & Prostate cancer & 5-Aza-CdR & $>100$ folds & {$[37]$} \\
\hline miR-21 & $\begin{array}{l}\text { PDCD4, TPM1, } \\
\text { and MARCKS }\end{array}$ & Prostate cancer & 5-Aza-CdR & $>2$ folds & {$[37]$} \\
\hline miR-370 & IRS-1 & Oral squamous cell carcinoma & 5-Aza-CdR & $2-20$ folds & {$[56]$} \\
\hline miR-373 & MBD2 & Hilar cholangiocarcinoma & 5-Aza-CdR or 5-Aza-CdR + TSA & $2-5$ folds & {$[57]$} \\
\hline miR-193b & ETS1, CCND1, PLAU & Prostate cancer & 5-Aza-CdR + TSA & $>2$ folds & [58] \\
\hline miR-132 & HB-EGF,TALIN2 & Prostate cancer & 5-Aza-CdR or 5-Aza-CdR + TSA & $>5$ folds & [59] \\
\hline miR-145 & BNIP3, TNFSF10,PAK7 & $\begin{array}{l}\text { Prostate cancer and clear } \\
\text { cell renal cell carcinomas }\end{array}$ & 5-Aza-CdR or 5-Aza-CdR + TSA & $>2$ folds & {$[53,60]$} \\
\hline miR-141, miR-514 & $\begin{array}{l}\text { HGF, CDC42, } \\
\text { RBX1, SLC2A1 }\end{array}$ & Clear cell renal cell carcinomas & 5-Aza-CdR + TSA & $>2$ folds & [53] \\
\hline miR-335 & $\mathrm{SOX} 4, \mathrm{Rb} 1$ & Hepatocellular carcinoma & 5-Aza-CdR + TSA & $>2$ folds & [61] \\
\hline miR-205 & $B C L-w$ & Prostate cancer & 5-Aza-CdR & $2-5$ folds & {$[62]$} \\
\hline let-7 family & RAS & Nasopharyngeal carcinoma & Zebularine & 2-100 folds & [63] \\
\hline \multicolumn{6}{|l|}{ Up-regulated } \\
\hline $\operatorname{miR}-224$ & API-5 & Hepatocellular carcinoma & C646 & $<50 \%$ & {$[36]$} \\
\hline miR-17-92 Cluster & PTEN, BCL2L11, CDKN1A & Colorectal Cancer & SAHA or TSA & $25 \%-67 \%$ & [64] \\
\hline
\end{tabular}

DNMT inhibitors: 5-azacytidine (5-AzaC); 5-aza-2'-deoxycytidine(5-Aza-CdR); Zebularine.

HDAC inhibitors: suberoylanilide hydroxamic acid (SAHA); trichostatin A (TSA); panobinostat(LBH589); phenylbutyrate (PBA); OSU-HDAC42(AR-42).

HAT inhibitors: C646.

HMT inhibitors: 3-deazaneplanocin A (DZNep).

altered owning to histone modification. This is partially due to the lack of effective detection methods and relatively strict requirements for obtaining and examining clinical samples.

Notably, the expanding role of MYC in epigenetics has recently received further attention. First, MYC can promote the expression of epigenetic enzymes, which contribute to tumor development. For example, MYC can induce the accumulation of EZH2 in prostate cancer [66].
Second, recent evidence attributed the deregulated miRNA expression to MYC, which is involved in promoting oncogenic miRNAs and repressing tumor suppressor miRNAs $[67,68]$. Considering the known mechanisms of histone modification, MYC might function as an initiator of miRNA epigenetic silencing, which can recruit enzymatic effectors such as HDAC and EZH2 to the miRNA promoter. Conversely, HDT and HAT are rarely reported in miRNA regulation, pointing out the needing to evaluate 
the potential of epigenetic drugs to re-express or repress deregulated miRNAs that contribute to carcinogenesis. Owing to the reversible nature of epigenetic alterations, therapeutic strategies targeting specific miRNAs based on epigenetic intervention might provide innovative tools for cancer treatment in the future. Further understanding of epigenetic mechanisms in miRNA regulation along with the effect of epigenetic drugs on specific miRNAs might help to reset the abnormal cancer epigenome.

\begin{abstract}
Abbreviations
DNMTs: DNA methyltransferases; BCL: B-cell lymphomas; HDACs: Histone deacetylases; EZH2: Enhancer of zeste homolog 2; YY-1: Yin and yang 1; NF-kB: Nuclear factor kB; RMS: Rhabdomyosarcoma; IkBa: Inhibitor of nuclear factor KB a; CLL: Chronic lymphocytic leukemia; MCL: Mantle cell lymphoma; ATL: Adult T cell leukemia; API-5: Apoptosis inhibitor-5; HCC: Hepatocellular carcinoma; TSA: Trichostatin A; AML: Acute myeloid leukemia; HATs: Histone acetyltransferases; HMTs: Histone methyltransferases; HDTs: Histone demethylases; LSD1: Lysine-specific demethylase 1; 5-AzaC: 5-azacytidine; 5-Aza-CdR: 5-aza-2'- deoxycytidine; MDS: Myelodysplastic syndromes; SAHA: Suberoylanilide hydroxamic acid; CTCL: Cutaneous T-cell lymphoma; LBH589: Panobinostat; PBA: Phenylbutyrate; AR-42: OSU-HDAC42; DZNep: 3-deazaneplanocin A.
\end{abstract}

\section{Competing interests}

The authors declare that they have no competing interests.

\section{Authors' contributions}

$X L L$ and $Y C$ were the main authors of the manuscript; XYC and XFY contributed to bibliography collection as well as figures and tables design and format; YGT revised the manuscript for important intellectual content; $A B$ corrected the language form; ZGD was responsible for the manuscript writing and sequence alignment. All authors read and approved the final manuscript.

\section{Acknowledgments}

This project was supported by the National Basic Research Program of China (2009CB522300), the National Nature Science Foundation of China (90813028 and 30830113) and Hunan Provincial Innovation Foundation for Postgraduate.

\section{Author details}

${ }^{1}$ Cancer Research Institute, Central South University, Changsha, Hunan 410078, China. ${ }^{2}$ Key Laboratory of Carcinogenesis and Cancer Invasion, Ministry of Education, Changsha, Hunan 410078, China. ${ }^{3}$ Key Laboratory of Carcinogenesis, Ministry of Health, Changsha, Hunan 410078, China. ${ }^{4}$ Molecular Imaging Center, Central South University, Changsha, Hunan 410078, China. ${ }^{5}$ The Hormel Institute, University of Minnesota, 801 16th Avenue NE, Austin, MN 55912, USA.

Received: 21 October 2013 Accepted: 14 November 2013 Published: 22 November 2013

\section{References}

1. Calin GA, Sevignani C, Dumitru CD, Hyslop T, Noch E, Yendamuri S, Shimizu M, Rattan S, Bullrich F, Negrini M: Human microRNA genes are frequently located at fragile sites and genomic regions involved in cancers. Proc Natl Acad Sci U S A 2004, 101:2999-3004.

2. Suzuki H, Takatsuka S, Akashi H, Yamamoto E, Nojima M, Maruyama R, Kai M, Yamano H-o, Sasaki Y, Tokino T: Genome-wide profiling of chromatin signatures reveals epigenetic regulation of microRNA genes in colorectal cancer. Cancer Res 2011, 71:5646-5658.

3. Iorio MV, Piovan C, Croce CM: Interplay between microRNAs and the epigenetic machinery: an intricate network. Biochim Biophys Acta Gene Regul Mech 2010, 1799:694-701

4. Lee K-H, Lotterman C, Karikari C, Omura N, Feldmann G, Habbe N, Goggins MG, Mendell JT, Maitra A: Epigenetic silencing of MicroRNA miR-107 regulates cyclin-dependent kinase 6 expression in pancreatic cancer. Pancreatology 2009, 9:293-301.

5. Saito Y, Liang G, Egger G, Friedman JM, Chuang JC, Coetzee GA, Jones PA: Specific activation of microRNA-127 with downregulation of the proto-oncogene BCL6 by chromatin-modifying drugs in human cancer cells. Cancer Cell 2006, 9:435-443.

6. Kunej T, Godnic I, Ferdin J, Horvat S, Dovc P, Calin GA: Epigenetic regulation of microRNAs in cancer: an integrated review of literature. Mutat Res 2011, 717:77-84

7. Laird PW: Principles and challenges of genome-wide DNA methylation analysis. Nat Rev Genet 2010, 11:191-203.

8. Wiklund ED, Kjems J, Clark SJ: Epigenetic architecture and miRNA: reciprocal regulators. Epigenomics 2010, 2:823-840.

9. Fabbri M, Garzon R, Cimmino A, Liu Z, Zanesi N, Callegari E, Liu S, Alder H, Costinean S, Fernandez-Cymering C: MicroRNA-29 family reverts aberrant methylation in lung cancer by targeting DNA methyltransferases $3 \mathrm{~A}$ and 3B. Proc Natl Acad Sci 2007, 104:15805-15810.

10. Zhang X, Zhao X, Fiskus W, Lin J, Lwin T, Rao R, Zhang Y, Chan JC, Fu K, Marquez VE: Coordinated silencing of MYC-mediated miR-29 by HDAC3 and $\mathrm{EZH} 2$ as a therapeutic target of histone modification in aggressive B-cell lymphomas. Cancer Cell 2012, 22:506-523.

11. Wilkinson FH, Park K, Atchison ML: Polycomb recruitment to DNA in vivo by the YY1 REPO domain. Proc Natl Acad Sci 2006, 103:19296-19301.

12. Wang $X$, Feng $Y, X u L$, Chen $Y$, Zhang $Y$, Su D, Ren G, Lu J, Huang B: YY1 restrained cell senescence through repressing the transcription of p16. Biochim Biophys Acta 1876, 2008:1783.

13. Ren G, Zhang G, Dong Z, Liu Z, Li L, Feng Y, Su D, Zhang Y, Huang B, Lu J: Recruitment of HDAC4 by transcription factor $Y Y 1$ represses HOXB13 to affect cell growth in AR-negative prostate cancers. Int J Biochem Cell Biol 2009, 41:1094-1101.

14. Wang H, Garzon R, Sun H, Ladner KJ, Singh R, Dahlman J, Cheng A, Hall BM, Qualman SJ, Chandler DS: NF-KB-YY1-miR-29 regulatory circuitry in skeletal myogenesis and rhabdomyosarcoma. Cancer Cell 2008, 14:369-381.

15. Liu S, Wu L-C, Pang J, Santhanam R, Schwind S, Wu Y-Z, Hickey CJ, Yu J, Becker $\mathrm{H}$, Maharry K, et al: Sp1/NFkB/HDAC/miR-29b regulatory network in KIT-driven myeloid leukemia. Cancer Cell 2010, 17:333-347.

16. Sampath D, Liu C, Vasan K, Sulda M, Puduvalli VK, Wierda WG, Keating MJ: Histone deacetylases mediate the silencing of miR-15a, miR-16, and miR-29b in chronic lymphocytic leukemia. Blood 2012, 119:1162-1172.

17. Johnson SM, Grosshans H, Shingara J, Byrom M, Jarvis R, Cheng A, Labourier E, Reinert $\mathrm{KL}$, Brown D, Slack FJ: RAS is regulated by the let-7 MicroRNA family. Cell 2005, 120:635-647.

18. Hayashi Y, Tsujii M, Wang J, Kondo J, Akasaka T, Jin Y, Li W, Nakamura T, Nishida T, lijima H: CagA mediates epigenetic regulation to attenuate let-7 expression in Helicobacter pylori-related carcinogenesis. Gut 2013, 62:1536-1546.

19. Brueckner B, Stresemann C, Kuner R, Mund C, Musch T, Meister M, Sültmann H, Lyko F: The human let-7a-3 locus contains an epigenetically regulated microRNA gene with oncogenic function. Cancer Res 2007, 67:1419-1423.

20. Calin GA, Dumitru CD, Shimizu M, Bichi R, Zupo S, Noch E, Aldler H, Rattan S, Keating M, Rai K: Frequent deletions and down-regulation of micro-RNA genes miR15 and miR16 at 13q14 in chronic lymphocytic leukemia. Proc Natl Acad Sci 2002, 99:15524-15529.

21. Wendtner CM: Cocktail of eternity: HDAC meets miR. Blood 2012 119:1095-1096

22. Zhang $X$, Chen X, Lin J, Lwin T, Wright G, Moscinski LC, Dalton WS, Seto E, Wright K, Sotomayor E, Tao J: Myc represses miR-15a/miR-16-1 expression through recruitment of HDAC3 in mantle cell and other non-Hodgkin B-cell lymphomas. Oncogene 2011, 31:3002-3008.

23. Ivanov SV, Goparaju CM, Lopez P, Zavadil J, Toren-Haritan G, Rosenwald S, Hoshen M, Chajut A, Cohen D, Pass HI: Pro-tumorigenic effects of miR-31 loss in mesothelioma. J Biol Chem 2010, 285:22809-22817.

24. Asangani IA, Harms PW, Dodson L, Pandhi M, Kunju LP, Maher CA, Fullen DR, Johnson TM, Giordano TJ, Palanisamy N: Genetic and epigenetic loss of microRNA-31 leads to feed-forward expression of EZH2 in melanoma. Oncotarget 2012, 3:1011-1025.

25. Augoff K, McCue B, Plow EF, Sossey-Alaoui K: miR-31 and its host gene IncRNA LOC554202 are regulated by promoter hypermethylation in triple-negative breast cancer. Mol Cancer 2012, 11:5.

26. Yamagishi M, Nakano K, Miyake A, Yamochi T, Kagami $Y$, Tsutsumi A Matsuda Y, Sato-Otsubo A, Muto S, Utsunomiya A: Polycomb-mediated loss of miR-31 activates NIK-dependent NF-KB pathway in adult T cell leukemia and other cancers. Cancer Cell 2012, 21:121.

27. Gao P, Tchernyshyov I, Chang TC, Lee YS, Kita K, Ochi T, Zeller Kl, De Marzo AM, Van Eyk JE, Mendell JT: c-Myc suppression of miR-23a/b enhances 
mitochondrial glutaminase expression and glutamine metabolism. Nature 2009, 458:762-765

28. Rathore MG, Saumet A, Rossi J-F, De Bettignies C, Tempé D, Lecellier C-H, Villalba M: The NF-kB member p65 controls glutamine metabolism through miR-23a. Int J Biochem Cell Biol 2012, 44:1448-1456.

29. Witt O, Deubzer HE, Milde T, Oehme I: HDAC family: what are the cancer relevant targets? Cancer Lett 2009, 277:8-21.

30. Au SLK, Wong CCL, Lee JMF, Fan DNY, Tsang FH, Ng IOL, Wong CM: Enhancer of zeste homolog 2 epigenetically silences multiple tumor suppressor microRNAs to promote liver cancer metastasis. Hepatology 2012, 56:622-631.

31. Buurman $R$, Gürlevik E, Schäffer V, Eilers M, Sandbothe M, Kreipe H, Wilkens L, Schlegelberger B, Kühnel F, Skawran B: Histone deacetylases activate hepatocyte growth factor signaling by repressing MicroRNA-449 in hepatocellular carcinoma cells. Gastroenterology 2012, 143:811-820. e815.

32. Cao Q, Mani R-S, Ateeq B, Dhanasekaran SM, Asangani IA, Prensner JR, Kim JH, Brenner JC, Jing X, Cao X: Coordinated regulation of polycomb group complexes through microRNAs in cancer. Cancer Cell 2011, 20:187-199.

33. Bao B, Ali S, Banerjee S, Wang Z, Logna F, Azmi AS, Kong D, Ahmad A, Li Y Padhye S: Curcumin analogue CDF inhibits pancreatic tumor growth by switching on suppressor microRNAs and attenuating EZH2 expression. Cancer Res 2012, 72:335-345.

34. Mitra D, Das PM, Huynh FC, Jones FE: Jumonji/ARID1 B (JARID1B) protein promotes breast tumor cell cycle progression through epigenetic repression of microRNA let-7e. J Biol Chem 2011, 286:40531-40535.

35. Wang Y, Lee AT, Ma JZ, Wang J, Ren J, Yang Y, Tantoso E, Li K-B, Ooi LL, Tan P: Profiling microRNA expression in hepatocellular carcinoma reveals microRNA-224 up-regulation and apoptosis inhibitor-5 as a microRNA224-specific target. J Biol Chem 2008, 283:13205-13215.

36. Wang Y, Toh HC, Chow P, Chung AY, Meyers DJ, Cole PA, Ooi LL, Lee CG: MicroRNA-224 is up-regulated in hepatocellular carcinoma through epigenetic mechanisms. FASEB J 2012, 26:3032-3041.

37. Hulf T, Sibbritt T, Wiklund ED, Bert S, Strbenac D, Statham AL, Robinson MD, Clark SJ: Discovery pipeline for epigenetically deregulated miRNAs in cancer: integration of primary miRNA transcription. BMC Genomics 2011, 12:54.

38. Greither T, Grochola LF, Udelnow A, Lautenschläger C, Würl P, Taubert H: Elevated expression of microRNAs 155, 203, 210 and 222 in pancreatic tumors is associated with poorer survival. Int J Cancer 2010, 126:73-80.

39. Jiang S, Zhang H-W, Lu M-H, He X-H, Li Y, Gu H, Liu M-F, Wang E-D: MicroRNA-155 functions as an OncomiR in breast cancer by targeting the suppressor of cytokine signaling 1 gene. Cancer Res 2010, 70:3119-3127.

40. Chang S, Wang R-H, Akagi K, Kim K-A, Martin BK, Cavallone L, Haines DC, Basik M, Mai P, Poggi E, et al: Tumor suppressor BRCA1 epigenetically controls oncogenic microRNA-155. Nat Med 2011, 17:1275-1282.

41. Börno ST, Fischer A, Kerick M, Fälth M, Laible M, Brase JC, Kuner R, Dahl A, Grimm C, Sayanjali B: Genome-wide DNA methylation events in TMPRSS2-ERG fusion-negative prostate cancers implicate an EZH2-dependent mechanism with miR-26a hypermethylation. Cancer Discov 2012, 2:1024-1035.

42. Balaguer F, Link A, Lozano JJ, Cuatrecasas M, Nagasaka T, Boland CR, Goel A: Epigenetic silencing of miR-137 is an early event in colorectal carcinogenesis. Cancer Res 2010, 70:6609-6618.

43. Datta J, Kutay H, Nasser MW, Nuovo GJ, Wang B, Majumder S, Liu C-G, Volinia S, Croce CM, Schmittgen TD: Methylation mediated silencing of MicroRNA-1 gene and its role in hepatocellular carcinogenesis. Cancer Res 2008, 68:5049-5058.

44. Garzon R, Liu S, Fabbri M, Liu Z, Heaphy CEA, Callegari E, Schwind S, Pang J, Yu J, Muthusamy N: MicroRNA-29b induces global DNA hypomethylation and tumor suppressor gene reexpression in acute myeloid leukemia by targeting directly DNMT3A and 3B and indirectly DNMT1. Blood 2009, 113:6411-6418.

45. Azmi AS, Beck FW, Bao B, Mohammad RM, Sarkar FH: Aberrant epigenetic grooming of miRNAs in pancreatic cancer: a systems biology perspective. Epigenomics 2011, 3:747-759.

46. Cortez CC, Jones PA: Chromatin, cancer and drug therapies. Mut Res/ Fundam Mol Mech Mutagen 2008, 647:44-51.

47. Boumber $Y$, Issa J: Epigenetics in cancer: what's the future. Oncology 2011, 25:220-226

48. Rodríguez-Paredes M, Esteller M: Cancer epigenetics reaches mainstream oncology. Nat Med 2011, 17:331.
49. Marks PA, Breslow R: Dimethyl sulfoxide to vorinostat: development of this histone deacetylase inhibitor as an anticancer drug. Nat Biotechnol 2007, 25:84-90.

50. Yang $X$, Feng M, Jiang X, Wu Z, Li Z, Aau M, Yu Q: miR-449a and miR-449b are direct transcriptional targets of E2F1 and negatively regulate $\mathrm{PRb}-\mathrm{E} 2 \mathrm{~F} 1$ activity through a feedback loop by targeting CDK6 and CDC25A. Genes Dev 2009, 23:2388-2393.

51. Alpini G, Glaser SS, Zhang JP, Francis H, Han Y, Gong J, Stokes A, Francis T, Hughart N, Hubble L: Regulation of placenta growth factor by microRNA-125b in hepatocellular cancer. J Hepatol 2011, 55:1339-1345.

52. Saito Y, Friedman JM, Chihara Y, Egger G, Chuang JC, Liang G: Epigenetic therapy upregulates the tumor suppressor microRNA-126 and its host gene EGFL7 in human cancer cells. Biochem Biophys Res Commun 2009, 379:726-731.

53. Wotschofsky Z, Liep J, Meyer H-A, Jung M, Wagner I, Disch AC, Schaser KD, Melcher I, Kilic E, Busch J: Identification of metastamirs as metastasisassociated MicroRNAs in clear cell renal cell carcinomas. Int J Biol Sci 2012, 8:1363-1374

54. Lodygin D, Tarasov V, Epanchintsev A, Berking C, Knyazeva T, Körner H, Knyazev P, Diebold J, Hermeking H: Inactivation of miR-34a by aberrant CpG methylation in multiple types of cancer. Cell Cycle 2008, 7:2591-2600.

55. Lujambio A, Calin G, Villanueva A, Ropero S, Sánchez-Céspedes M, Blanco D, Montuenga L, Rossi S, Nicoloso M, Faller W: A microRNA DNA methylation signature for human cancer metastasis. Proc Natl Acad Sci U S A 2008, 105:13556-13561.

56. Chang K, Chu T, Gong N, Chiang W, Yang C, Liu C, Wu C, Lin S: miR-370 modulates insulin receptor substrate-1 expression and inhibits the tumor phenotypes of oral carcinoma. Oral Dis 2013, 19:611-619.

57. Chen Y, Gao W, Luo J, Tian R, Sun H, Zou S: Methyl-CpG binding protein MBD2 is implicated in methylation-mediated suppression of miR-373 in hilar cholangiocarcinoma. Oncol Rep 2011, 25:443.

58. Rauhala HE, Jalava SE, Isotalo J, Bracken H, Lehmusvaara S, Tammela TL, Oja H, Visakorpi T: miR-193b is an epigenetically regulated putative tumor suppressor in prostate cancer. Int J Cancer 2010, 127:1363-1372.

59. Formosa A, Lena A, Markert E, Cortelli S, Miano R, Mauriello A, Croce N, Vandesompele J, Mestdagh P, Finazzi-Agrò E: DNA methylation silences miR-132 in prostate cancer. Oncogene 2012, 32:127-134.

60. Zaman M, Chen Y, Deng G, Shahryari V, Suh S, Saini S, Majid S, Liu J, Khatri G, Tanaka Y: The functional significance of microRNA-145 in prostate cancer. Br J Cancer 2010, 103:256-264.

61. Dohi O, Yasui K, Gen Y, Takada H, Endo M, Tsuji K, Konishi C, Yamada N, Mitsuyoshi $\mathrm{H}$, Yagi N: Epigenetic silencing of miR-335 and its host gene MEST in hepatocellular carcinoma. Int J Oncol 2013, 42:411-418.

62. Bhatnagar N, Li X, Padi S, Zhang Q, Tang M, Guo B: Downregulation of miR-205 and miR-31 confers resistance to chemotherapy-induced apoptosis in prostate cancer cells. Cell Death Dis 2010, 1:e105.

63. Wong T-S, Man O-Y, Tsang C-M, Tsao S-W, Tsang RK-Y, Chan JY-W, Ho W-K, Wei WI, To VS-H: MicroRNA let-7 suppresses nasopharyngeal carcinoma cells proliferation through downregulating c-Myc expression. J Cancer Res Clin Oncol 2011, 137:415-422

64. Humphreys KJ, Cobiac L, Le Leu RK, Van der Hoek MB, Michael MZ: Histone deacetylase inhibition in colorectal cancer cells reveals competing roles for members of the oncogenic miR-17-92 cluster. Mol Carcinog 2013, 52:459-474

65. Cao Y, DePinho RA, Ernst M, Vousden K: Cancer research: past, present and future. Nat Rev Cancer 2011, 11:749-754.

66. Koh CM, Iwata T, Zheng Q, Bethel C, Yegnasubramanian S, De Marzo AM: Myc enforces overexpression of EZH2 in early prostatic neoplasia via transcriptional and post-transcriptional mechanisms. Oncotarget 2011, 2:669.

67. Chang T-C, Yu D, Lee Y-S, Wentzel EA, Arking DE, West KM, Dang CV, Thomas-Tikhonenko A, Mendell JT: Widespread microRNA repression by Myc contributes to tumorigenesis. Nat Genet 2007, 40:43-50.

68. Sander S, Bullinger L, Klapproth K, Fiedler K, Kestler HA, Barth TF, Möller P, Stilgenbauer S, Pollack JR, Wirth T: MYC stimulates EZH2 expression by repression of its negative regulator miR-26a. Blood 2008, 112:4202-4212.

doi:10.1186/1756-9966-32-96

Cite this article as: Liu et al:: Regulation of microRNAs by epigenetics and their interplay involved in cancer. Journal of Experimental \& Clinical Cancer Research 2013 32:96. 